\title{
HIV status, age at cervical Cancer screening and cervical cytology outcomes in an opportunistic screening setting in Nigeria: a 10-year Cross sectional data analysis
}

Jonah Musa ${ }^{1,2,3^{*}}$ (D), Chad J. Achenbach ${ }^{3,4}$, Charlesnika T. Evans ${ }^{5,6}$, Neil Jordan ${ }^{6,7}$, Patrick H. Daru', Olugbenga Silas ${ }^{8}$, Atiene S. Sagay ${ }^{1}$, Rose Anorlu' ${ }^{9}$, Supriya D. Mehta ${ }^{10}$, Firas Wehbe ${ }^{11}$, Melissa A. Simon ${ }^{12}$, Isaac F. Adewole ${ }^{13}$, Lifang $\mathrm{Hou}^{3,14}$ and Robert L. Murphy,

\begin{abstract}
Background: Invasive cervical cancer (ICC) is more prevalent in HIV infected women and occurs at younger median age than in HIV negative women. Organized cervical cancer screening (CCS) is presently lacking in Nigeria, and the age at CCS is not known in this population. We sought to examine the age at CCS, the cytology outcomes and whether outcomes differ by HIV infection status in an opportunistic screening setting.

Methods: Cross-sectional analysis of data on a sample of women who had received a CCS in an opportunistic screening service in Jos, Nigeria over a 10-year time period (2006-2016). We used logistic regression models to estimate the independent effect of patient-reported HIV and age at CCS and odds ratios for abnormal cytology outcomes adjusting for other covariates. We also assessed the correlation between median age at CCS and severity of abnormal cervical cytology outcomes. Statistical analyses were done on STATA version 14, College Station, Texas, USA.
\end{abstract}

Results: In a sample of 14,088, the median age at CCS was 37 years (IQR; 30-45). For HIV infected women vs. uninfected women, CCS occurred at earlier ages (35.0 \pm 7.4 vs $38.2 \pm 10.2$ years, $p<0.001)$. All women, regardless of HIV status, who completed at least 7 or more years of education were 1.27 to 3.51 times more likely to have CCS before age 35 than women with less education. The predictors of an abnormal cervical cytology outcome at CCS were: age at $\mathrm{CCS} \geq 35(\mathrm{aOR}=3.57 ; 95 \% \mathrm{Cl}: 2.74,4.64)$, multiparity $\geq 5(\mathrm{aOR}=1.27 ; 95 \% \mathrm{Cl}: 1.03,1.56)$, and providerreferral (aOR $=1.34 ; 95 \% \mathrm{Cl}: 1.09,1.64)$. Irrespective of reported HIV status, we found a positive correlation between median age at CCS and severity of cytology outcome.

Discussion: The age at CCS in women who have utilized cervical cancer screening in the study population is relatively late compared to the recommended age by most guidelines from developed settings. Late age at CCS correlates positively with severity of abnormal cytology outcome irrespective of HIV status. More educated women are more likely to have CCS at early age and less likely to have underlying abnormal cytology outcomes.

Keywords: Cervical cancer screening, HIV status, Age at screening, Opportunistic screening, Cytology outcome, Nigeria

\footnotetext{
*Correspondence: jonahmusa2009@u.northwestern.edu;

Jonah.musa@northwestern.edu; drmusaj@yahoo.com; jonahm@unijos.edu.ng

'Department of Obstetrics and Gynecology, College of Health Sciences,

University of Jos, Jos, Plateau, Nigeria

${ }^{2}$ Department of Preventive Medicine, Division of Cancer Epidemiology and

Prevention, Feinberg School of Medicine, Northwestern University, Chicago,

IL, USA

Full list of author information is available at the end of the article
}

(c) The Author(s). 2019 Open Access This article is distributed under the terms of the Creative Commons Attribution 4.0 International License (http://creativecommons.org/licenses/by/4.0/), which permits unrestricted use, distribution, and reproduction in any medium, provided you give appropriate credit to the original author(s) and the source, provide a link to the Creative Commons license, and indicate if changes were made. The Creative Commons Public Domain Dedication waiver (http://creativecommons.org/publicdomain/zero/1.0/) applies to the data made available in this article, unless otherwise stated. 


\section{Introduction}

Even though invasive cervical cancer (ICC) is a preventable cancer, there are a half million new cases of ICC reported globally each year, with over $80 \%$ occurring in LMICs [1]. In Nigeria, over 53 million women are estimated to be at risk of ICC, and available cervical cancer screening (CCS) covers less than 9\% of the population [2]. This lack of CCS availability contributes to approximately 14,000 new cases and 8000 deaths attributed to ICC every year [2]. The Global Burden of Cancer 2013 ranked ICC the 2nd most common in incidence and mortality for all cancers in Nigeria [3].

CCS is an important health care intervention for reducing ICC incidence and mortality with substantial benefits recorded in developed countries, where organized CCS programs are available [4-10]. In Nigeria the high prevalence of HIV [11] and the lack of organized CCS programs are substantial contributing factors to the high burden of ICC. In settings where organized CCS programs are lacking, the opportunity to have a screening test depends on several factors including the availability of a screening service and system support to overcome barriers to accessing such services; patient-related factors such as risk perception for ICC, illiteracy, and lack of awareness of CCS, or lack of knowledge and access to such screening [12-15]. Other important factors include cost of screening, health insurance coverage, education, perception of screening benefits and ability to overcome barriers to accessing services [13, 16].

Since the aim of CCS is to prevent cervical cancer through identification and treatment of precancerous cervical lesions, understanding the socio-demographic factors associated with abnormal cervical cytology outcomes could provide evidence for educating women and providers on the benefits of screening, particularly in women with certain characteristics. These predictors could also guide development of country-level screening guidelines for CCS and prevention. For instance, a French healthcare database on CCS provided evidence for not starting screening before age 25 [17], in comparison to the United States Preventive Service Task Force (USPSTF) guideline [18, 19] that recommends starting CCS at age 21 .

Of particular interest are the findings from previous reports in sub-Saharan Africa that ICC is not only more prevalent in HIV infected women but occurs at a lower median age of 35 years compared to a median age of 40 years in women who are HIV negative [20]. Also, among women less than age 35 , being HIV positive confers a 4fold higher risk of having ICC compared to being HIV negative [20]. Therefore, HIV infected women may benefit from CCS by screening at relatively younger ages compared to HIV seronegative. Yet data from a large CCS program in Zambia, showed that the median age at first CSS was higher in HIV seropositive women compared to HIV seronegative women, reflecting that evidence related to HIV status, CCS, and ICC is not incorporated in the implementation of CCS [21].

In addition to the lack of an organized CCS program and poor coverage for available screening services, the age at CCS is not known in Nigeria [2]. We also do not know the effectiveness of screening in terms of the likelihood of detecting an underlying abnormal cervical cytology at the time of screening. In this paper, we sought to examine the age at CCS, the cytology outcomes and whether these outcomes differ by HIV status in an opportunistic screening setting in Nigeria.

\section{Methods}

\section{Study design, setting and sample population}

The detail of the study design, setting and sample derivation for this cross-sectional analysis has been described previously [22]. In brief, we utilized de-identified data of 14,088 women who had received a CCS at the "Operation Stop Cervical Cancer '(OSCC) Unit in, Jos, Nigeria, over a 10-year time period (2006-2016). We accessed the reported age at CCS and other relevant sociodemographic variables, risk factors, self-reported HIV status and the cytology outcomes reported by the cytopathologist. The cervical Pap cytology screening outcomes were reported according to the Bethesda 2001 cytology reporting system [23]. The primary independent variable for this analysis was self-reported HIV status at the time of CCS. The key dependent variables were age at CCS and the cytology outcome (see Additional file 1 for sample derivation and the dependent variables in the analysis of this manuscript). The description of the key variables and the cytology outcomes are provided in Additional file 2.

\section{Statistical analysis \\ Descriptive statistics}

We performed summary statistics on continuous and categorical variables of the study sample and obtained means, medians and proportions for the independent and dependent variables. We also compared the baseline characteristics of the sample with the primary outcome. The Student's t-test was used to assess differences in means of normally distributed continuous variables by HIV status. In this analysis, women who did not know their HIV status were treated as missing.

\section{Analysis for age at $\mathrm{CCS}<35$ years as primary outcome}

Since the principal exposure variable in this analysis was patient-reported HIV status, we estimated the mean age of women who received a CCS by patient-reported HIV status. We performed the Student's t-test of differences in means between two groups (mean age of women who 
were "HIV infected" as group 1, and mean age of women who were HIV uninfected as group 2. Based on previous literature showing that the median age at developing ICC was 35 years in HIV infected women [20], we dichotomized the age at CCS in our sample at $<35$ years and $\geq 35$ years. We compared the baseline socio-demographic characteristics of the sample by age at CCS $<35$ years compared to $\geq 35$ years.

\section{Bivariable and multivariable logistic regression model}

To further understand the independent effect of patientreported HIV on the age at CCS, we performed bivariable logistic regression analysis using various demographic variables as independent variables and dichotomizing the age at CCS as either $<35$ years as the primary outcome " 1 " or age CCS $\geq 35$ years as the referent category "0". We also created dummy variables for other socio-demographic variables such as smoking, alcohol, years of completed education $(<7$ years as group $1,7-12$ years as group 2 and $>12$ years as group 3 ), history of ever been diagnosed with an STI, age at first coitus, history of vaginal infection, total lifetime number of sex partners, parity, and provider-referral. We first performed a bivariable logistic regression on each of these reported characteristics with age at CCS $<35$ years as the primary outcome. We then used a multivariable logistic regression model to assess the independent predictive effect of patient-reported HIV on the likelihood of having a CCS at age $<35$ years in our cervical cancer screening population. We used the backward selection method to build our final predictive model. We estimated $95 \%$ confidence intervals for each of these measures of association and corresponding $p$-values.

\section{Analysis for abnormal cytology outcome at CCS as primary outcome}

We estimated the relative proportions of the various categories of pap cytology outcomes at CCS reported according to the Bethesda system and the corresponding 95\% CI. The median age at CCS for each of the cytology outcome categories and the corresponding interquartile range (IQR) were estimated. For analytic convenience and ease of interpretation we categorized the cytology report into three groups as follows: negative for intraepithelial lesion or malignancy (NILM) as category 1 (referent category); ASCUS and LSIL (mild cervical dysplasia) as category 2; and ASC-H, AGUS, HSIL, HSIL with suspicion for invasion (severe cervical dysplasia) as category 3 . We also estimated the proportions for each of these sub-categories. We compared the baseline socio-demographic characteristics of the study sample by cervical cytology groups using the Pearson's chi square or Fisher's exact test where applicable and obtained corresponding $p$-values.

\section{Bivariable logistic regression}

We performed bivariable logistic regression to obtain the odds ratios of the association between baseline sociodemographic variables and abnormal cervical cytology, dummy variables were created for each of the cytology outcome categories with category 1 (NILM) as referent. We then performed separate bivariable logistic regression to estimate the likelihood of having mild cervical dysplasia (category 2) and severe cervical dysplasia (category 3) respectively at CCS for self-reported HIV and other sociodemographic characteristics in the study sample. For each of these categories, we estimated the unadjusted odds ratio, $95 \%$ CIs, and the corresponding $p$-values.

\section{Multivariable logistic regression}

We built a multivariable logistic regression model to assess the independent effect of self-reported HIV and other socio-demographic characteristics on the likelihood of an abnormal cervical cytology outcome report at CCS. As in the bivariable logistic regression model, we used category 1 cytology report (NILM) as referent. We then performed separate multivariable logistic regression models each for mild cervical dysplasia (category 2) and for severe cervical dysplasia (category 3). We used the backward selection method with $p<0.05$ and the overall changes in the model effect to select the covariates that remain in each of the final predictive models. We estimated the $95 \%$ confidence intervals for each of these measures of association, and the corresponding $p$-values. The assessment of each model fit was by the Hosmer-Lemeshow goodnes's-of-fit statistical test [24]. A p-value of greater than 0.05 was considered a good model-fit.

\section{Results}

During the study period 14,054 out of the 14,088 (99.8\%) women reported the age at CCS, while 14,081 (99.95\%) women had cervical cytology reports. The proportion of women who screened at $<35$ years was significantly higher for women who were HIV infected (51.5\%) compared to women who were HIV uninfected $(40.2 \%)(p<0.001)$. The mean age at CCS for HIV infected women was $35.0 \pm 7.4$ years compared to $38.2 \pm$ 10.2 years for HIV uninfected women $(p$-value $=0.001$ ). The Boxplot in Fig. 1 showed a significant difference in the age at CCS for HIV infected women compared to HIV uninfected. The results of the analyses for age at CCS have been summarized in Tables 1 and 2 .

The baseline sociodemographic and cytology outcomes of the study sample has been published in an earlier related report and shown in Table 3 [22]. In brief, $85.7 \%$ of the study sample had NILM, while 9.7 and $4.6 \%$ had mild and severe cervical dysplasia respectively. Specifically, 4.1\% (95\% CI: 3.8, 4.5\%) with ASCUS, 5.6\% (95\% CI: 5.3, 6.0) with LSIL, $1.6 \%$ (95\% CI: $1.4,1.8)$ with ASC-H, $0.2 \%$ (95\% CI: 


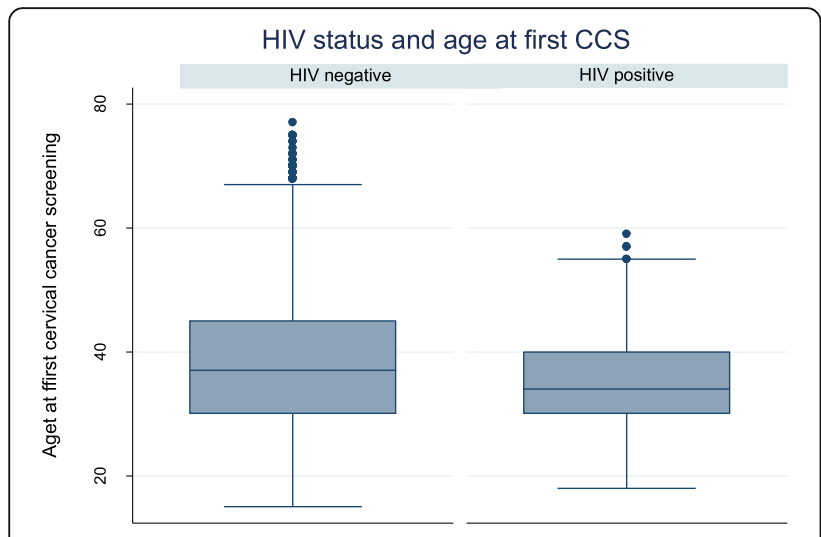

Fig. 1 Box Plot of Age at CCS by patient-reported HIV status

$0.2,0.3)$ with AGUS, $2.5 \%$ (95\% CI: $2.3,2.8$ ) with HSIL, and $0.2 \%$ (95\% CI, $0.2,0.3$ ) with HSIL with suspicion for invasion. The median age for the various cytology categories were: 36 years (IQR; 30-43) for NILM, 43 years (IQR; 3650) for ASCUS, 45 years (IQR; 35-52) for LSIL, 47.5 years (IQR; 38-55) for ASCUS-H, 40 years (95\% CI, 34-52) for
AGUS, 47 years (IQR; 39-55) for HSIL, and 52 years (IQR; 43-60) for HSIL with suspicion for invasion. The scatter plot in Fig. 2 of the median age at CCS and the predicted cytology outcome category reflects a positive linear relationship between median age and severity of cytology outcome at cervical screening $\left(\mathrm{r}=0.31\right.$; Adj. $\mathrm{R}^{2}=0.47 ; p$ value $=0.054$ ). Self-reported HIV status was not significantly associated with mild or severe cervical dysplasia in the study sample $(p$-value $=0.930)$. The association between other socio-demographic variables with cervical cytology outcomes are displayed in Table 4.

\section{Unadjusted and adjusted logistic regression model of self-reported HIV and other sociodemographic variables and mild cervical dysplasia}

In the unadjusted regression model, self-reported HIV infection was not significantly associated with mild cervical dysplasia $(\mathrm{OR}=0.99 ; 95 \% \mathrm{CI}: 0.77,1.28)$. The sociodemographic factors that were significantly associated with mild cervical dysplasia were: age at CCS $\geq 35$ years $(\mathrm{OR}=2.83 ; 95 \% \mathrm{CI}: 2.48,3.24)$, multiparity $\geq 5(\mathrm{OR}=$

Table 1 Baseline socio-demographic characteristics by age at first CCS $<35$ years versus $\geq 35$ years in an opportunistic screening program in Jos, Nigeria $(N=14,051)$

\begin{tabular}{|c|c|c|c|}
\hline Variable & Age first CCS $\geq 35$ years & Age first CCS $<35$ years & p-value \\
\hline HIV status & & & $0.001^{\dagger}$ \\
\hline Not infected & $7870(59.8)$ & $5285(40.2)$ & \\
\hline Infected & $341(48.5)$ & $362(51.5)$ & \\
\hline Age at first CCS (Mean \pm SD) & $8305(44.5 \pm 7.7)$ & $5749(22.7 \pm 3.7)$ & $0.001^{\neq}$ \\
\hline \multicolumn{4}{|l|}{ No of Life-time sex } \\
\hline partners(Mean \pm SD) & $6185(2.2 \pm 1.9)$ & $5104(2.2 \pm 1.8)$ & $0.503^{\ddagger}$ \\
\hline \multicolumn{4}{|l|}{ Use of condom } \\
\hline No & $7307(60.2)$ & $4838(39.8)$ & $0.001^{\dagger}$ \\
\hline Yes & $404(40.0)$ & $605(60.0)$ & \\
\hline \multicolumn{4}{|l|}{ History of smoking } \\
\hline No & $8222(59.3)$ & $5653(40.7)$ & $0.272^{\dagger}$ \\
\hline Yes & $42(53.2)$ & $37(46.8)$ & \\
\hline \multicolumn{4}{|l|}{ History of Alcohol } \\
\hline No & $7625(58.5)$ & $5410(41.5)$ & $0.001^{\dagger}$ \\
\hline Yes & $635(69.7)$ & $276(30.3)$ & \\
\hline \multicolumn{4}{|l|}{ History of vaginal infection } \\
\hline No & $1536(65.6)$ & $805(34.4)$ & $0.001^{\dagger}$ \\
\hline Yes & $6517(57.8)$ & $4756(42.2)$ & \\
\hline \multicolumn{4}{|l|}{ Ever diagnosed with STI } \\
\hline No & $4963(58.0)$ & $3598(42.0)$ & $0.001^{\dagger}$ \\
\hline Yes & $744(52.9)$ & $662(47.1)$ & \\
\hline Age at first sex (Mean $\pm S D$ ) & $8193(19.9 \pm 4.1)$ & $5651(20.4 \pm 3.8)$ & $0.001^{\neq}$ \\
\hline Education years completed (Mean \pm SD) & $6610(11.8 \pm 3.4)$ & $5117(11.9 \pm 2.6)$ & $0.062^{\ddagger}$ \\
\hline Parity (Mean \pm SD) & $7818(4.4 \pm 2.5)$ & $4317(2.1 \pm 1.7)$ & $0.001^{\ddagger}$ \\
\hline
\end{tabular}

${ }^{\text {F}}$ Student t-test and ${ }^{\dagger}$ Pearson's chi ${ }^{2}$. Percent in parenthesis, $S D$ standard deviation 
Table 2 Bivariable and multivariable Logistic regression model with unadjusted and adjusted odds ratio of the association between patient-reported HIV, other socio-demographic factors and the likelihood of first CCS at age $<35$ years in an opportunistic cervical cancer screening program in Jos, Nigeria $(N=14,051)$

\begin{tabular}{|c|c|c|c|c|}
\hline Variable & OR $(95 \% \mathrm{Cl})$ & $P$-value & $\mathrm{aOR}(95 \% \mathrm{Cl})$ & $P$-value \\
\hline \multicolumn{5}{|l|}{ HIV status } \\
\hline Not infected & 1.0 & & & \\
\hline Infected & $1.58(1.36,1.84)$ & 0.001 & $1.18(0.99,1.41)$ & 0.058 \\
\hline \multicolumn{5}{|l|}{ Referral group } \\
\hline Self-referral & 1.0 & 0.001 & - & - \\
\hline Provider-referral & $0.75(0.70,0.80)$ & & & \\
\hline \multicolumn{5}{|c|}{ Education (years completed) } \\
\hline$<7$ years & 1.0 & & & \\
\hline $7-12$ years & $3.12(2.75,3.53)$ & 0.001 & $3.07(2.69,3.51)$ & 0.001 \\
\hline$>12$ years & $1.53(1.36,1.72)$ & 0.001 & $1.43(1.27,1.62)$ & 0.001 \\
\hline \multicolumn{5}{|l|}{ Parity } \\
\hline$<5$ & 1.0 & & & \\
\hline$\geq 5$ & $0.51(0.47,0.55)$ & 0.001 & - & - \\
\hline \multicolumn{5}{|l|}{ Age at first sex } \\
\hline$>22$ years & 1.0 & & & \\
\hline$\leq 22$ years & $0.83(0.77,0.90)$ & 0.001 & - & - \\
\hline \multicolumn{5}{|c|}{ Total life-time sex partners } \\
\hline$<3$ & 1.0 & & & \\
\hline$\geq 3$ & $1.14(1.05,1.24)$ & 0.001 & - & - \\
\hline
\end{tabular}

Use of condoms during sex

$\begin{array}{lllll}\text { No } & 1.0 & & & \\ \text { Yes } & 2.26(1.98,2.58) & 0.001 & 1.96(1.70,2.27) & 0.001\end{array}$

History of vaginal infection

$\begin{array}{lllll}\text { No } & 1.0 & & & \\ \text { Yes } & 1.39(1.27,1.53) & 0.001 & 1.29(1.15,1.43) & 0.001\end{array}$

Ever diagnosed with STIS

$\begin{array}{lllll}\text { No } & 1.0 & & \\ \text { Yes } & 1.23(1.10,1.37) & 0.001 & - & \\ \text { History of Smoking } & (N=13,954) & & & \\ \text { No } & 1.0 & & & \\ \text { Yes } & 1.28(0.82,2.0) & 0.273 & 1.63(0.93,2.83) & 0.086 \\ \text { Alcohol consumption }(13,946) & & & \\ \text { No } & 1.0 & & - \\ \text { Yes } & 0.61(0.53,0.71) & 0.001 & -\end{array}$

The Hosmer-Lemeshow goodness-of-fit $p$-value $=0.538$, Pseudo $R^{2}=0.0363$, $L R\left(c h i^{2}\right)=521.35$

1.46; $95 \% \mathrm{CI}: 1.31,1.64)$, age at first sexual intercourse $\leq 22$ years $(\mathrm{OR}=1.23$; 95\% CI: 1.08, 1.41), providerreferral $(\mathrm{OR}=1.88 ; 95 \% \mathrm{CI}: 1.67,2.11)$, history of ever smoked cigarettes $(\mathrm{OR}=1.84 ; 95 \% \mathrm{CI}: 1.01,3.35)$ and history of alcohol consumption $(\mathrm{OR}=1.50$; 95\% CI: 1.23 ,
1.83). One notable finding in the unadjusted model is that women with 7 or more completed years of education were significantly less likely to have mild cervical dysplasia at CCS than women with less than 7 completed years of education $(7-12$ years, OR $=0.68$; 95\% CI: $0.56,0.84 ;>12$ years, $\mathrm{OR}=0.82 ; 95 \% \mathrm{CI}: 0.68,0.96$ ). These unadjusted ORs are presented in Table 5.

In the adjusted logistic regression model including age at $\mathrm{CCS} \geq 35$, provider-referral, multiparity $\geq 5$, history of vaginal infection and alcohol consumption, the effect of self-reported HIV infection was not significantly associated with mild cervical dysplasia $(\mathrm{aOR}=1.04 ; 95 \% \mathrm{CI}$ : $0.80,1.36)$. The sociodemographic variables that were independently associated with mild cervical dysplasia were: age at $\mathrm{CCS} \geq 35(\mathrm{aOR}=2.56 ; 95 \% \mathrm{CI}: 2.23,2.95)$, multiparity $\geq 5(\mathrm{aOR}=1.21 ; 95 \% \mathrm{CI}: 1.08,1.36)$, providerreferral $(\mathrm{aOR}=1.75 ; 95 \% \mathrm{CI}: 1.56,1.98)$ and history of alcohol consumption $(\mathrm{aOR}=1.38$; 95\% CI: 1.38 ; 95\% CI: $1.13,1.70)$. These adjusted ORs are presented in Table 5.

\section{Unadjusted and adjusted logistic regression model of self-reported HIV and other sociodemographic variables and severe cervical dysplasia}

In the unadjusted regression model, self-reported HIV infection was not significantly associated with severe cervical dysplasia $(\mathrm{OR}=0.93$; 95\% CI: $0.64,1.35)$. The sociodemographic factors that were significantly associated with severe cervical dysplasia were: age at CCS $\geq 35$ years $(\mathrm{OR}=4.24 ; 95 \% \mathrm{CI}: 3.40,5.29)$, multiparity $\geq 5$ (OR $=$ 1.85; 95\% CI: $1.58,2.17)$, age at first sexual intercourse $\leq 22$ years $(\mathrm{OR}=1.32$; 95\% CI: 1.08, 1.60), providerreferral $(\mathrm{OR}=1.27 ; 95 \% \mathrm{CI}: 1.08,1.49)$. Similar to the unadjusted model for mild dysplasia, women with 7-12 completed years or more of education were significantly less likely to have severe cervical dysplasia at CCS than women with less than 7 completed years of education ( $7-12$ years, OR $=0.46 ; 95 \% \mathrm{CI}: 0.34,0.62$; > 12 years, $\mathrm{OR}=0.63$; 95\% CI: $0.49,0.80)$. The unadjusted ORs are presented in Table 6.

In the adjusted logistic regression model including age at $\mathrm{CCS} \geq 35$, provider-referral, multiparity $\geq 5$, history of vaginal infection, 7-12 years of completed education, and $>12$ years of completed education, the effect of selfreported HIV infection was not significantly associated with severe cervical dysplasia $(\mathrm{aOR}=1.26 ; 95 \% \mathrm{CI}$ : 0.83 , 1.92). The sociodemographic variables that were independently associated with severe cervical dysplasia were: age at $\mathrm{CCS} \geq 35(\mathrm{aOR}=3.57$; 95\% CI: $2.74,4.64)$, multiparity $\geq 5$ ( $\mathrm{aOR}=1.27 ; 95 \% \mathrm{CI}: 1.03,1.56$ ), and providerreferral $(\mathrm{aOR}=1.34 ; 95 \% \mathrm{CI}: 1.09,1.64)$. Women with $7-12$ completed years of education $(\mathrm{aOR}=0.65 ; 95 \% \mathrm{CI}$ : $0.48,0.88),>12$ completed years of education $(\mathrm{aOR}=$ 0.75 ; $95 \%$ CI: $0.58,0.98)$, and history of vaginal infection $(\mathrm{aOR}=0.67 ; 95 \% \mathrm{CI}: 0.53,0.84)$ were significantly less 
Table 3 Summary statistics of the socio-demographic and cytology outcomes of women who received first CCS in an opportunistic cervical cancer screening program in Jos Nigeria $(N=14,088)$

\begin{tabular}{|c|c|c|}
\hline Characteristics & Descriptive statistics (Mean $\pm S D$, Median, IQR or \% in parentheses) & 95\% Confidence intervals \\
\hline Age at CCS & 37; IQR, 30-45 & \\
\hline \multicolumn{3}{|l|}{ Age groups at CCS } \\
\hline$<21$ years & 1.1 & $1.0,1.3$ \\
\hline $21-30$ & 24.7 & $24.0,25.4$ \\
\hline $31-40$ & 37.3 & $36.5,38.1$ \\
\hline $41-50$ & 25.4 & $24.6,26.1$ \\
\hline $51-60$ & 8.9 & $8.5,9.4$ \\
\hline $61-70$ & 2.1 & $1.8,2.3$ \\
\hline$\geq 71$ & 0.2 & $0.2,0.3$ \\
\hline Missing & 0.2 & $0.2,0.3$ \\
\hline Age at first sex & 20; IQR, 18-22 & \\
\hline Education years completed & $13 ; I Q R, 12-14$ & \\
\hline Annual household income in USD & 3300; IQR, 1920-4800 & \\
\hline \multicolumn{3}{|l|}{ HIV status } \\
\hline Infected & $703(5.0)$ & $4.6-5.5$ \\
\hline Not infected & $13,155(93.4)$ & $93.0-93.8$ \\
\hline Unknown (missing) & $230(1.6)$ & $1.4-1.9$ \\
\hline \multicolumn{3}{|l|}{ History of Vaginal infection } \\
\hline Yes & 80.0 & $79.4-80.7$ \\
\hline No & 16.6 & $16.0-17.2$ \\
\hline Missing & 3.4 & $3.1-3.7$ \\
\hline \multicolumn{3}{|l|}{ Use of condoms } \\
\hline Yes & 7.4 & $6.8-7.6$ \\
\hline No & 86.2 & $85.6-86.8$ \\
\hline Missing & 6.6 & $6.2-7.1$ \\
\hline \multicolumn{3}{|l|}{ Ever diagnosed with an STI } \\
\hline Yes & 10.0 & $9.5-10.5$ \\
\hline No & 60.8 & $60.0-61.6$ \\
\hline Missing & 29.3 & $28.5-30.0$ \\
\hline \multicolumn{3}{|l|}{ Types of STls } \\
\hline Gonorrhea & 17.0 & $14.0-20.5$ \\
\hline Trichomonads & 6.7 & $4.8-9.2$ \\
\hline Hepatitis & 40.5 & $36.4-44.8$ \\
\hline Chlamydia & 28.7 & $17.3-47.1$ \\
\hline HPV/Genital warts & 5.9 & $4.2-8.3$ \\
\hline Syphilis & 4.8 & $3.3-7.0$ \\
\hline Herpes & 3.4 & $2.2-5.4$ \\
\hline PID/Unspecified & 18.3 & $15.6-22.3$ \\
\hline \# of Lifetime sex partners & $2 ; I Q R, 1-3$ & \\
\hline Parity & $3 ; \mid \mathrm{QR}, 2-3$ & \\
\hline \multicolumn{3}{|l|}{ History of smoking } \\
\hline Yes & 0.6 & $0.5-0.7$ \\
\hline No & 98.5 & $98.3-98.7$ \\
\hline
\end{tabular}


Table 3 Summary statistics of the socio-demographic and cytology outcomes of women who received first CCS in an opportunistic cervical cancer screening program in Jos Nigeria $(N=14,088)$ (Continued)

\begin{tabular}{|c|c|c|}
\hline Characteristics & Descriptive statistics (Mean \pm SD, Median, IQR or \% in parentheses) & 95\% Confidence intervals \\
\hline Missing & 1.0 & $0.8-1.1$ \\
\hline \multicolumn{3}{|l|}{ History of Alcohol } \\
\hline Yes & 6.5 & $6.1-6.9$ \\
\hline No & 92.5 & $92.1-93.0$ \\
\hline missing & 1.0 & $0.9-1.2$ \\
\hline \multicolumn{3}{|l|}{ Race } \\
\hline Black & 99.7 & $99.6-99.8$ \\
\hline Others & 0.1 & $0.1-0.2$ \\
\hline Missing & 0.2 & $0.1-0.30$ \\
\hline \multicolumn{3}{|l|}{ Cytology outcome at CCS } \\
\hline NILM & 85.7 & $85.1-86.3$ \\
\hline ASCUS & 4.1 & $3.8-4.5$ \\
\hline LSIL & 5.6 & $5.3-6.0$ \\
\hline $\mathrm{ASC}-\mathrm{H}$ & 1.6 & $1.4-1.8$ \\
\hline AGUS & 0.2 & $0.2-0.3$ \\
\hline HSIL & 2.5 & $2.3-2.8$ \\
\hline HSIL, suspicion for invasion & 0.2 & $0.2-0.3$ \\
\hline \multicolumn{3}{|l|}{ Cytology category at CCS } \\
\hline Normal cervical cytology & 85.7 & $85.1-86.3$ \\
\hline Mild cervical dysplasia & 9.7 & $9.3-10.2$ \\
\hline Severe cervical dysplasia & 4.6 & $4.2-4.9$ \\
\hline
\end{tabular}

SD standard deviation, IQR Interquartile range, \% (Percent)

likely to have severe cervical dysplasia at first CCS. These adjusted ORs are presented in Table 6.

\section{Discussion}

The results of our analyses have contributed to our understanding of socio-demographic factors associated with utilization and cytology screening outcomes in an

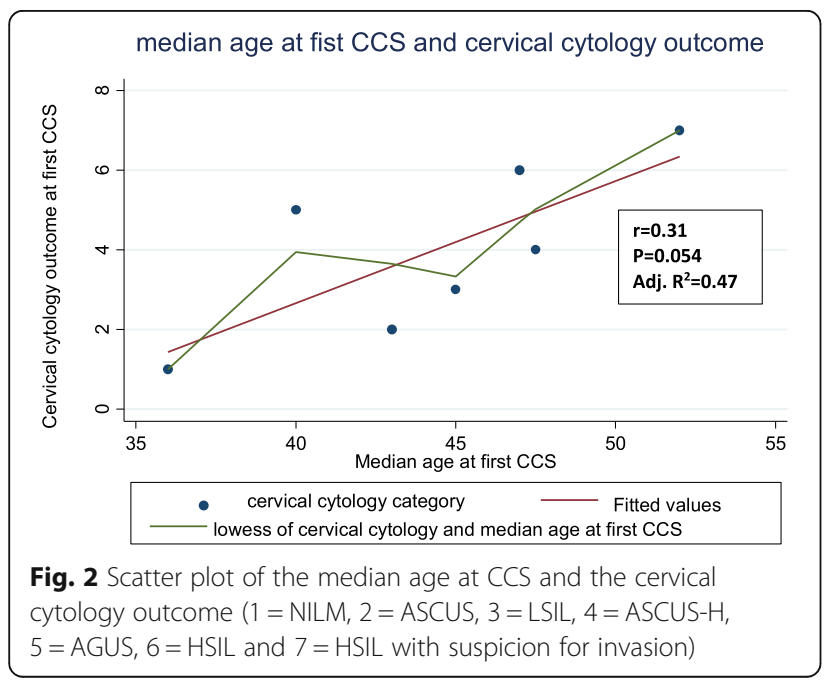

opportunistic CCS program in Jos, Nigeria. We found that women who had utilized the opportunistic CCS service in the population had screening at a median age of 37 years (IQR 30-45). We also found that on average, women who were HIV infected had CCS at a younger age than women who were HIV uninfected. Also, women who completed at least 7 years of education were 1.27 to 3.51 times more likely to have had CCS before age 35 than women with less education.

Our study findings have significant implications for cervical cancer prevention and screening in Nigeria. The median age at CCS is relatively late at 37 years, and this is of concern for cervical cancer prevention and control given the evidence that ICC occurs at a median age of 35 years in HIV infected women, 40 years in HIV uninfected women, and 38 years in women with unknown HIV status [20]. The relatively late screening age in our sample suggests that many women may have already developed precancerous conditions of the cervix or invasive cancer at the time of CCS. This finding could also explain the high rate of advanced stage ICC with high death rates as reported in previous studies [25-28].

Related to the age at CCS, an earlier study report from a district hospital in Abuja, Nigeria's federal capital, 
Table 4 Baseline socio-demographic characteristics by cervical cytology category at CCS in an opportunistic screening program in Jos, Nigeria $(N=14,081)$

\begin{tabular}{|c|c|c|c|c|}
\hline Variable & NILM & Mild Dysplasia & Severe dysplasa & $p$-value \\
\hline \multicolumn{5}{|l|}{ HIV status } \\
\hline Not infected & $11,261(85.7)$ & $1288(9.8)$ & $599(4.6)$ & \multirow[t]{2}{*}{$0.930^{\mathrm{a}}$} \\
\hline Infected & $605(86.1)$ & $68(9.7)$ & $30(4.3)$ & \\
\hline \multicolumn{5}{|l|}{ Age at CCS } \\
\hline$<35$ years & $5367(93.4)$ & $288(5.0)$ & $94(1.6)$ & \multirow[t]{2}{*}{$0.001^{t}$} \\
\hline$\geq 35$ years & $6701(80.4)$ & $1083(13.0)$ & $548(6.6)$ & \\
\hline \multicolumn{5}{|c|}{ Total \# lifetime sex partners } \\
\hline$<3$ & $6727(85.5)$ & $763(9.7)$ & $374(4.8)$ & \multirow[t]{2}{*}{$0.001^{a}$} \\
\hline$\geq 3$ & $3035(88.7)$ & $271(7.9)$ & $114(3.3)$ & \\
\hline \multicolumn{5}{|l|}{ Use of condom } \\
\hline No & $10,436(86.0)$ & $1166(9.6)$ & $540(4.4)$ & \multirow[t]{2}{*}{$0.002^{a}$} \\
\hline Yes & $904(89.5)$ & $81(8.0)$ & $25(2.5)$ & \\
\hline \multicolumn{5}{|c|}{ History of smoking } \\
\hline No & $11,899(85.8)$ & $1340(9.7)$ & $630(4.5)$ & \multirow[t]{2}{*}{$0.145^{\mathrm{b}}$} \\
\hline Yes & $63(79.8)$ & $13(16.4)$ & $3(3.8)$ & \\
\hline \multicolumn{5}{|c|}{ History of Alcohol } \\
\hline No & $11,212(86.1)$ & $1230(9.4)$ & $588(4.5)$ & \multirow[t]{2}{*}{$0.001^{a}$} \\
\hline Yes & $743(81.7)$ & $123(13.5)$ & $44(4.8)$ & \\
\hline \multicolumn{5}{|c|}{ History of vaginal infection } \\
\hline No & $1919(82.0)$ & $276(11.8)$ & $145(6.2)$ & \multirow[t]{2}{*}{$0.001^{a}$} \\
\hline Yes & 9752 (86.6) & $1036(9.2)$ & $480(4.2)$ & \\
\hline \multicolumn{5}{|c|}{ Ever diagnosed with STI } \\
\hline No & $7431(86.8)$ & $763(8.9)$ & $365(4.3)$ & \multirow[t]{2}{*}{$0.843^{\mathrm{a}}$} \\
\hline Yes & $1228(87.3)$ & $122(8.7)$ & $56(4.0)$ & \\
\hline \multicolumn{5}{|l|}{ Age at first sex } \\
\hline$\geq 22$ years & $8025(84.5)$ & $996(10.5)$ & $476(5.0)$ & \multirow[t]{2}{*}{$0.001^{a}$} \\
\hline$<22$ years & $3843(88.5)$ & $345(8.0)$ & $153(3.5)$ & \\
\hline \multicolumn{5}{|c|}{ Education years completed } \\
\hline$<7$ years & $1366(83.8)$ & $172(10.5)$ & $93(5.7)$ & \multirow[t]{3}{*}{$0.001^{a}$} \\
\hline $7-12$ years & $3078(89.8)$ & $256(7.5)$ & $93(2.7)$ & \\
\hline$>12$ years & $5834(87.6)$ & $584(8.8)$ & $244(3.6)$ & \\
\hline \multicolumn{5}{|l|}{ Parity } \\
\hline$<5$ & $7382(88.2)$ & $699(8.4)$ & $288(3.4)$ & \multirow[t]{2}{*}{$0.001^{a}$} \\
\hline$\geq 5$ & $2894(77.0)$ & $563(14.9)$ & $303(8.1)$ & \\
\hline
\end{tabular}

${ }^{\mathrm{a}}$ Pearson's chi ${ }^{2}$. ${ }^{\mathrm{F}}$ Fisher's Exact. Percent in parenthesis

found a mean age of $32.0 \pm 6.6$ years at first CCS by visual inspection with acetic acid (VIA) [29]. Compared to the mean age of $35.0 \pm 7.4$ years at first CCS in our study sample, the slightly lower mean age at first screening in the Abuja HIV population could partly be explained by the mode of screening using VIA, and the specific program intervention, which involved active interaction between HIV infected women receiving antiretroviral
Table 5 Bivariable and multivariable Logistic regression with unadjusted and adjusted odds ratio of the association of patient-reported HIV and other sociodemographic variables and mild cervical dysplasia at CCS in Jos, Nigeria $(N=13,554)$

\begin{tabular}{lllll}
\hline Variable & OR $(95 \% \mathrm{Cl})$ & $P$-value & aOR $(95 \% \mathrm{Cl})$ & $P$-value \\
\hline HIV status & & & & \\
$\quad$ Uninfected & 1.0 & & & \\
$\quad$ Infected & $0.99(0.77,1.28)$ & 0.953 & $1.04(0.80,1.36)$ & 0.747 \\
Age at CCS & & & & \\
$\quad<35$ years & 1.0 & & & \\
$\geq 35$ years & $2.83(2.48,3.24)$ & 0.001 & $2.56(2.23,2.95)$ & 0.001 \\
Referral group & & & & \\
$\quad \begin{array}{l}\text { Self-referral } \\
\text { Provider-referral }\end{array}$ & 1.0 & & & \\
& $1.88(1.67,2.11)$ & 0.001 & $1.75(1.56,1.98)$ & 0.001
\end{tabular}

Education (years completed)

$\begin{array}{llll}<7 \text { years } & 1.0 & & \\ 7-12 \text { years } & 0.68(0.56,0.84) & 0.001 & - \\ >12 \text { years } & 0.82(0.68,0.96) & 0.025 & -\end{array}$

Parity

$<5 \quad 1.0$

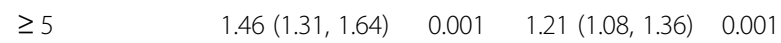

Age at first sex

$\begin{array}{cccc}>22 \text { years } & 1.0 & \\ \leq 22 \text { years } & 1.23(1.08,1.41) & 0.002 & - \\ \text { Total life-time sex partners } & \\ <3 & 1.0 & \\ \geq 3 & 0.80(0.69,0.93) & 0.003 & -\end{array}$

Use of condoms during sex

$$
\begin{array}{ccccc}
\text { No } & 1.0 & & \\
\text { Yes } & 0.82(0.65,1.04) & 0.103 & - \\
\text { History of vaginal infection } & & & \\
\text { No } & 1.0 & & & \\
\text { Yes } & 0.76(0.69,0.87) & 0.001 & 0.81(0.70,0.94) & 0.004
\end{array}
$$

Ever diagnosed with STIS

No $\quad 1.0$

Yes $\quad 0.97(0.79,1.19) \quad 0.772 \quad-$

History of Smoking

$$
\begin{array}{llll}
\text { No } & 1.0 & & \\
\text { Yes } & 1.84(1.01,3.35) & 0.045 & -
\end{array}
$$

Alcohol consumption

\begin{tabular}{lllll} 
No & 1.0 & & & \\
Yes & $1.50(1.23,1.83)$ & 0.001 & $1.38(1.13,1.70)$ & 0.002 \\
\hline
\end{tabular}

Hosmer-Lemeshow Goodnes-of-fit $p$-value $=0.145$, LR (chi2) $=365.90$,

Pseudo $\mathrm{R}^{2}=0.0425$

therapy and provider-initiated CCS with VIA during the intervention period [29]. VIA is technically less sophisticated than cytology-based screening which is usually 
Table 6 Bivariable and multivariable Logistic regression with unadjusted and adjusted odds ratio of the association of patient-reported HIV and other sociodemographic variables and severe cervical dysplasia at CCS in Jos, Nigeria $(N=11,345)$

\begin{tabular}{|c|c|c|c|c|}
\hline Variable & OR $(95 \% \mathrm{Cl})$ & $P$-value & $\mathrm{aOR}(95 \% \mathrm{Cl})$ & $P$-value \\
\hline \multicolumn{5}{|l|}{ HIV status } \\
\hline Uninfected & 1.0 & & & \\
\hline Infected & $0.93(0.64,1.35)$ & 0.704 & $1.26(0.83,1.92)$ & 0.276 \\
\hline
\end{tabular}

Age at first CCS

$<35$ years $\quad 1.0$

$\begin{array}{lllll}\geq 35 \text { years } \quad 4.24(3.40,5.29) & 0.001 & 3.57(2.74,4.64) & 0.001\end{array}$

Referral group

Self-referral $\quad 1.0$

Provider-referral $1.27(1.08,1.49) \quad 0.004 \quad 1.34(1.09,1.64) \quad 0.005$

Education (years completed)

$\begin{array}{lllll}<7 \text { years } & 1.0 & & & \\ \text { 7-12 years } & 0.46(0.34,0.62) & 0.001 & 0.65(0.48,0.88) & 0.006 \\ >12 \text { years } & 0.63(0.49,0.80) & 0.001 & 0.75(0.58,0.98) & 0.034\end{array}$

Parity

$<5 \quad 1.0$

$\begin{array}{lllll}\geq 5 & 1.85(1.58,2.17) & 0.001 & 1.27(1.03,1.56) & 0.025\end{array}$

Age at first sex

$\begin{array}{llll}>22 \text { years } & 1.0 & & \\ \leq 22 \text { years } & 1.32(1.08,1.60) & 0.006 & - \\ & & & \\ \text { Total lifetime sex partners } & 1.0 & & \\ <3 & 0.69(0.56,0.86) & 0.001 & -\end{array}$

Use of condoms during sex

$\begin{array}{lllll}\text { No } & 1.0 & & \\ \text { Yes } & 0.55(0.36,0.82) & 0.004 & - \\ \text { History of vaginal infection } & & & \\ \text { No } & 1.0 & & & \\ \text { Yes } & 0.67(0.56,0.82) & 0.001 & 0.67(0.53,0.84) & 0.001\end{array}$

Ever diagnosed with STIS

$\begin{array}{clll}\text { No } & 1.0 & & \\ \text { Yes } & 0.93(0.70,1.24) & 0.627 & - \\ \text { History of Smoking } & & \end{array}$

$\begin{array}{lll}\text { No } & 1.0 & \\ \text { Yes } & 0.83(0.26,2.64) & 0.751 \quad-\end{array}$

Alcohol consumption

$\begin{array}{llll}\text { No } & 1.0 & \\ \text { Yes } & 1.08(0.79,1.47) & 0.651 \quad-\end{array}$

Hosmer-Lemeshow Goodnesss-of-fit $p$-value 0.798. LR $\left(\mathrm{chi}^{2}\right)$-178.15, Pseudo $\mathrm{R}^{2}=0.0497$

done in tertiary health care facilities with cytopathologic laboratory support. Moreover, cytology-based screening methods have been shown to be more specific in detecting cervical precancer in HIV infected populations irrespective of immune status and antiretroviral treatment [30]. Overall, the findings on age at screening in our study population have broadened our knowledge and understanding of the current situation on CCS services in Nigeria and the need to leverage these data for health policy advocacy at state's and federal ministries of health to guide prevention efforts particularly the availability and access to screening either by cytology-based or "see-and-treat" by VIA as recommended by WHO for early detection and treatment of cervical precancerous conditions.

We also analyzed the association between selfreported HIV and abnormal cervical cytology outcome at CCS in our study sample. We found that self-reported HIV was not significantly associated with having either mild or severe cervical dysplasia at the time of first CCS. The weak association between HIV and abnormal cervical cytology outcome could be partly explained by the wide-spread use of highly active antiretroviral therapy (HAART) in our study sample. Also, this was a crosssectional data analysis with no follow up element to ascertain the risk of incident cervical abnormalities in HIV infected compared to women who were HIV negative. However, follow up data in a US population did not find a significant difference in incident cervical dysplasia and cancer in HIV population on successful HAART $[31,32]$. However, we found that women who had CCS at age $\geq 35$ years were 2.6 and 3.6 times more likely to have an underlying mild and severe cervical dysplasia, respectively. The utility of HSIL for early detection of cervical cancer has been studied in older women and its sensitivity for cancer was $89 \%$ in women screened at age $40-69$ and $83 \%$ in women screened at age $\geq 70$ years [33] Therefore, our study findings showing a severe dysplasia rate of $4.6 \%$ and that older age is a significant predictor of underlying severe dysplasia are useful findings that could contribute to developing and implementing CCS policy and guidelines with respect to age at which to start CCS in Nigeria.

A closer assessment of the relationship between age at CCS and abnormal cervical cytology outcome showed a positive correlation between median age at CCS and the severity of underlying cervical cytologic abnormality (Fig. 2). Though the strength of this correlation is modest with a borderline statistical significance $(\mathrm{r}=0.31 ; p=$ 0.054 and adjusted $R^{2}=0.47$ ), the median age at diagnosis of these abnormalities and the corresponding interquartile range suggest that implementing a Nigerian CCS policy and guidelines that cover screening between age 30 and 60 years may be an effective screening recommendation. Although our data are limited to one federal academic tertiary medical institution in northern Nigeria, a subsequent cost-effectiveness analysis can 
characterize and add to understanding the value of extending CCS outside this age range in the Nigerian population. Such understanding is crucial in resourceconstrained settings where health insurance coverage is limited. If subsequent cost-effectiveness analysis support screening within this age range, health policy makers could implement health insurance coverage for CCS for women ages 30 to 60 in Nigeria. However, there is need to obtain more large-scale screening data across the country to increase the precision of these estimates.

Our analyses also found that multiparity $\geq 5$ was significantly associated with mild or severe cervical dysplasia at first CCS. Specifically, women with parity $\geq 5$ were 1.85 and 1.27 times more likely to have an underlying mild or severe cervical dysplasia, respectively, at the time of first CCS. Studies on the cofactors in cervical precancer and progression to ICC have provided evidence that women of parity 3 or more were significantly more likely to have pre-cancer compared to nulliparous women [34]. In Nigeria, according to the Nigeria Demographic Health Survey (NDHS) 2013, the national average number of births per woman is 5.5 [35]. In many settings in sub-Saharan Africa and Nigeria, women place a high premium on parity, and this socio-cultural norm amidst poor coverage for CCS services could contribute to the burden of pre-cancer and ICC [36]. Other sociodemographic characteristics such as smoking, sexually transmitted infections, life-time number of sexual partners, and age at first sexual intercourse have been identified as significant cofactors in cervical carcinogenesis [37]. These identified cofactors associated with abnormal cervical cytology outcomes at CCS further provide justification for the prioritization of CCS services targeting women with these identified characteristics, particularly in settings where resources are limited.

We also found that women who were referred by providers for CCS were 1.34 times more likely to have severe cervical dysplasia outcome compared to women who self-referred for CCS. The plausibility of this finding is not fully understood though it may be related to the role of providers in identifying women with risk factors for cervical cancer, or observing cervical abnormalities or presentation with symptoms, and offering selective referral for screening in this population. Related to this, we have previously reported that women who received provider referral were more likely to be older and have known risk factors for cervical cancer [22]. Additionally, more educated women were more likely to utilize available CCS services at relatively younger age [22].

Our analysis further confirms the role of women's education in improving CCS utilization and outcomes. We found that completing at least 7-12 years of education significantly reduces the odds for severe cervical dysplasia by 25 to $35 \%$ compared to women who had fewer years of completed education. These findings are supported by previous studies showing the positive impact of educating women in improving cervical cancer outcomes [38, 39]. For instance, cervical cancer incidence and mortality are correlated with the sociodemographic index (SDI) of the population, with high SDI countries having a significantly lower ICC burden compared to low SDI countries [40]. In brief, the SDI ranges between 0 and 1 and is a summary indicator derived from measures of income per capita, educational attainment, and fertility [40]. An SDI of 1 represents a location with the highest observed educational attainment, the highest log income per capita, and the lowest fertility rate [40]. A previous related index, the human development index (HDI), which includes adult literacy rate and primary to tertiary education enrollment rates, has been shown to correlate inversely with incidence and mortality from ICC, with greater reductions in cervical cancer incidence in very high HDI compared to low HDI countries [41]. In Nigeria there is a wide regional disparity in median years of educational attainment, higher in the south-western states compared to the far north-east and north-western states [35]. The median years of educational attainment in the study area according to the NDHS 2013 report is 2.9 years [35]. The median years of educational attainment in our analytic sample was 13 years suggesting that only the more educated women utilized the available opportunistic CCS services and majority of the less educated in the larger population are either not aware or not able to overcome barriers to access the service. Our study therefore suggests the need for investment in developing and improving the educational status of women in our population as a social capital investment to improve cervical cancer outcomes. Added to this is the need for more cervical cancer education in low literacy communities to improve screening utilization [42].

The strength of our study findings is related to the relatively large sample size covering a 10 -year period in an opportunistic CCS in a cosmopolitan Nigerian city that also offers care to a large population of HIV infected adults in West Africa. To the best of our knowledge this is the first secondary analysis of CCS data in Nigeria that provides precise estimates of the age at CCS and the epidemiological factors associated with an underlying abnormal cervical cytology outcome. Because the women included in this analysis were self-selected having overcome barriers to accessing opportunistic CCS services, and may not be representative of the general population of women in Nigeria or West Africa, our findings are of limited external validity to other settings in Nigeria or West Africa amongst women with ongoing HIV care and amongst areas that have availability of opportunistic cervical cancer services. Also, self-reported 
risk factors such as age, age at first sex, life-time number of sex partners, use of condoms and HIV status is a limitation in this analysis. It is possible that some women may have concealed their HIV status and other sensitive socio-demographic information, and this could affect the internal validity of our estimates.

In conclusion, cervical cancer is a preventable cancer and organized CCS programs such as those in the industrialized nations have dramatically reduced incidence and mortality. Future research should include a focus on understanding provider and patient perspectives on the facilitators and barriers to CCS in an opportunistic screening setting using qualitative research methodology. However, our current findings could guide health policy leaders in the implementation of CCS guidelines particularly in our settings where CCS are largely opportunistic. Specifically, our findings of a relatively late age at first cervical cancer screening particularly in HIV infected women population will require a more focused effort and investment in awareness campaigns and cervical cancer education with emphasis on the benefits of starting screening at a younger age in order to maximize the overall gains of CCS as secondary preventive service for early detection and treatment of precancerous conditions. This remain an effective health service intervention for prevention of morbidity and mortality due to ICC in the population.

\section{Supplementary information}

Supplementary information accompanies this paper at https://doi.org/10. 1186/s13027-019-0263-4.

Additional file 1. Study sample derivation for study aims 1, 2 and 3 .

Note: the results presented in this manuscript are from aim 2 and primary aim 3.

Additional file 2. The operational definition of independent variables and the primary outcome.

\section{Acknowledgements}

The contributions of Joyce Asufi, RN, BNsc, and Patricia Asemota, RN, BNsc, of the Nursing department and "Operation Stop" cervical cancer unit of Jos University Teaching Hospital, Jos, Nigeria is hereby acknowledged. Mr. Clement Wambuda of the Records and Data Management unit of Jos University Teaching Hospital and "Operation Stop" cervical cancer unit, provided assistance with access to the electronic data files used in this analysis. Finally, the authors acknowledged Mr. Omar M. Dakwar, MSEB, of the Division of Infectious Diseases, Northwestern University, Chicago, USA for technical assistance with data management.

\section{Authors' contributions}

This work was conceptualized by JM, and refined by MAS, CTE, NJ, RLM and CJA. JM, PHD, OS, ASS, and IFA contributed to data acquisition for this analysis. JM performed statistical analyses with additional support and refinement of the analytic methods by CTE, NJ, SDM, FW, and CJA. JM produced the first draft of the manuscript with critical review, and interpretation of results by MAS, CTE, NJ, SDM, RA, PHD, ASS, IFA, OS, CJA, $\mathrm{LH}, \mathrm{FW}$, and RLM. All co-authors contributed to revising the manuscript and approved the final version for submission for publication.

\section{Funding}

This work and publication was supported by a training grant \#-D43TW009575 from the NIH Fogarty International Center and the National
Cancer Institute. This publication is partly supported by grant \# 1 U54 CA221205-01 from the National Cancer Institute, and a career development award from the NIH/Fogarty International Center, 1K43TW011416-01. The funding agencies cited here did not play a role in the design, collection of data, analysis, interpretation, and writing of this manuscript or decision to publish the results. The contents of this manuscript are solely the responsibility of the authors and do not necessarily represent the official views of $\mathrm{NIH}$ Fogarty International Center and the National Cancer Institute.

\section{Availability of data and materials}

All the relevant data for this analysis have been presented in the body of this manuscript. Additional information on the analytic sample have been provided in Additional files attached to this manuscript. The original data sources and the dataset used in this analysis is available upon reasonable request to the corresponding author.

\section{Ethics approval and consent to participate}

The Health Research and Ethics Committee of the Jos University Teaching Hospital, Jos, Nigeria approved the protocol of this study to use de-identified patient data for this analysis and provided a waiver of informed consent. Also, the Northwestern University Institutional Review Board, Northwestern University, Chicago, USA granted approval for this study and assigned a "non-human subject" determination for this analysis.

\section{Consent for publication}

Not applicable.

\section{Competing interests}

The authors declare that they have no competing interests.

\section{Author details}

${ }^{1}$ Department of Obstetrics and Gynecology, College of Health Sciences, University of Jos, Jos, Plateau, Nigeria. ${ }^{2}$ Department of Preventive Medicine, Division of Cancer Epidemiology and Prevention, Feinberg School of Medicine, Northwestern University, Chicago, IL, USA. ${ }^{3}$ Institute of Global Health, Feinberg School of Medicine, Northwestern University, Chicago, IL, USA. ${ }^{4}$ Division of Infectious Diseases, Department of Medicine, Feinberg School of Medicine, Northwestern University, Chicago, IL, USA. ${ }^{5}$ Department of Preventive Medicine, Center for Health Care Studies, Institute for Public Health and Medicine, Feinberg School of Medicine, Northwestern University, Chicago, IL, USA. ${ }^{6}$ Center of Innovation for Complex Chronic Healthcare (CINCCH), Department of Veterans Affairs, Edward Hines Jr. VA Hospital, Hines, IL, USA. Department of Psychiatry \& Behavioral Science, Feinberg School of Medicine, Northwestern University, Chicago, IL, USA. ${ }^{8}$ Department of Pathology, College of Health Sciences, University of Jos, Jos, Plateau State, Nigeria. ${ }^{9}$ Department of Obstetrics and Gynecology, College of Medicine, University of Lagos, Lagos, Lagos, Nigeria. ${ }^{10}$ Division of Epidemiology and Biostatistics, School of Public Health, University of Illinois at Chicago, Chicago, IL, USA. ${ }^{11}$ Department of Preventive Medicine, Division of Health and Biomedical Informatics, Feinberg School of Medicine, Northwestern University, Chicago, IL, USA. ${ }^{12}$ Department of Obstetrics and Gynecology, Preventive Medicine and Medical Social Sciences, Feinberg School of Medicine, Northwestern University, Chicago, IL, USA. ${ }^{13}$ Department of Obstetrics and Gynecology, College of Medicine, University of Ibadan, Ibadan, Oyo, Nigeria. ${ }^{14}$ Center for Population Epigenetics, Robert H. Lurie Comprehensive Cancer Center and Department of Preventive Medicine, Northwestern University Feinberg School of Medicine, Chicago, IL 60611, USA.

Received: 7 July 2019 Accepted: 20 November 2019

Published online: 29 November 2019

\section{References}

1. International Agency for Research on Cancer (IARC): Latest world cancer statistics Global cancer burden rises to 14.1 million new cases in 2012: Marked increase in breast cancers must be addressed. In. Edited by IARC. World Health Organization; Press Release No. 223; 2013. p. 1-3.

2. B-RL BL, AlberoG SB, Mena M, GómezD MJ, Bosch FX, de Sanjosé S. ICO/ IARC information Centre on HPV and Cancer (HPV information Centre). Human papillomavirus and related diseases in Nigeria. In: Summary Report 27 July 2017. ICO/IARC HPV Information Centre; 2017. 
3. Global Burden of Disease Cancer C, Fitzmaurice C, Dicker D, Pain A, Hamavid H, Moradi-Lakeh M, MF Ml, Allen C, Hansen G, Woodbrook R, et al. The global burden of Cancer 2013. JAMA oncol. 2015;1(4):505-27.

4. WHO: WHO guidelines for Screening and Treatment of Precancerous lesions for Cervical Cancer Prevention. WHO guidelines 2013.

5. White MC, Wong FL. Preventing premature deaths from breast and cervical cancer among underserved women in the United States: insights gained from a national cancer screening program. Cancer Causes Control. 2015;26(5):805-9.

6. Miller JW, Royalty J, Henley J, White A, Richardson LC. Breast and cervical cancers diagnosed and stage at diagnosis among women served through the National Breast and cervical Cancer early detection program. Cancer Causes Control. 2015;26(5):741-7.

7. Lantz PM, Mullen J. The National Breast and cervical Cancer early detection program: 25 years of public health service to low-income women. Cancer Causes Control. 2015;26(5):653-6.

8. Ekwueme DU, Uzunangelov VJ, Hoerger TJ, Miller JW, Saraiya M, Benard VB, Hall IJ, Royalty J, Li C, Myers ER. Impact of the National Breast and cervical Cancer early detection program on cervical cancer mortality among uninsured low-income women in the U.S., 1991-2007. Am J Prev Med. 2014;47(3):300-8.

9. Allemani C, Weir HK, Carreira H, Harewood R, Spika D, Wang X-S, Bannon F, Ahn JV, Johnson CJ, Bonaventure A, et al. Global surveillance of cancer survival 1995-2009: analysis of individual data for 25676887 patients from 279 population-based registries in 67 countries (CONCORD-2). Lancet. 2015; 385(9972):977-1010.

10. Moshkovich O, Lebrun-Harris L, Makaroff L, Chidambaran P, Chung M, Sripipatana A, Lin SC. Challenges and opportunities to improve cervical Cancer screening rates in US health centers through patient-centered medical home transformation. Adv Prev Med. 2015;2015:182073.

11. Bashorun A, Nguku P, Kawu I, Ngige E, Ogundiran A, Sabitu K, Nasidi A, Nsubuga P. A description of HIV prevalence trends in Nigeria from 2001 to 2010: what is the progress, where is the problem? Pan Afr Med J. 2014;18(Suppl 1):3

12. Idowu A, Olowookere SA, Fagbemi AT, Ogunlaja OA. Determinants of cervical Cancer screening uptake among women in Ilorin. J Cancer Epidemiol. 2016;2016:6469240.

13. Ndikom CM, Ofi BA. Awareness, perception and factors affecting utilization of cervical cancer screening services among women in Ibadan. Reprod Health. 2012;9:11.

14. Mfon S, Cyrus-David RM, Paskett ED, Dxgostino R Jr, Goff D. Cervical Cancer risk as a predictor of pap smear use in rural North Carolina. J Rural Health. 2002:8(1):67-76.

15. Ackerson K, Preston SD. A decision theory perspective on why women do or do not decide to have cancer screening: systematic review. J Adv Nurs. 2009;65(6):1130-40.

16. Shannon N, Westin DB, Gano JB, Fields MM, Coker AL, Sun CC, Ramondetta LM. Social factors affecting treatment of cervical Cancer: ethical issues and policy implications. Obstet Gynecol. 2008;111:747-51.

17. Maura G, Chaignot C, Weill A, Alla F, Heard I. Cervical cancer screening and subsequent procedures in women under the age of 25 years between 2007 and 2013 in France: a nationwide French healthcare database study. Eur J Cancer Prev. 2018;27:479-85.

18. USPSTF: USPSTF current recommendation for cervical Cancer screening. 2012.

19. Moyer VA. Screening for Cervical Cancer: U.S. Preventive Services Task Force Recommendation Statement. Ann Intern Med. 2012;156:880-91.

20. Kapambwe S, Sahasrabuddhe W, Blevins M, Mwanahamuntu MH, Mudenda V, Shepherd BE, Chibwesha CJ, Pfaendler KS, Hicks ML, Vermund SH, et al. Implementation and operational research: age distribution and determinants of invasive cervical Cancer in a "screen-and-treat" program integrated with HIV/AIDS Care in Zambia. J Acquir Immune Defic Syndr. 2015;70(1):e20-6.

21. Mwanahamuntu MH, Sahasrabuddhe W, Blevins M, Kapambwe S, Shepherd BE, Chibwesha C, Pfaendler KS, Mkumba G, Vwalika B, Hicks ML, et al. Utilization of cervical cancer screening services and trends in screening positivity rates in a 'screen-and-treat' program integrated with HIV/AIDS care in Zambia. PLoS One. 2013;8(9):e74607.

22. Musa J, Achenbach CJ, Evans CT, Jordan N, Daru PH, Hou L, Murphy RL, Adewole IF, Simon MA. Association between patient-reported HIV status and provider recommendation for screening in an opportunistic cervical Cancer screening setting in Jos. BMC Health Serv Res. 2018;18(1):885.

23. Solomon D, Davey D, Kurman R, Moriarty A, O'Connor D, Prey M, Raab S, Sherman M, Wilbur D, Wright T Jr, Young N; Forum Group Members; Bethesda 2001 Workshop. The 2001 Bethesda System: terminology for reporting results of cervical cytology. JAMA. 2002;287(16):2114-9.
24. Hosmer D, Hosmer T, Le Cessie S, Lemeshow S. A comparison of goodnessof-fit tests for the logistic regression model. Stat Med. 1997;16(9):965-80.

25. Maranga IO, Hampson L, Oliver AW, Gamal A, Gichangi P, Opiyo A, Holland CM, Hampson IN. Analysis of factors contributing to the low survival of cervical cancer patients undergoing radiotherapy in Kenya. PLoS One. 2013;8(10):e78411.

26. Yvonne Nartey PCH, Amo-Antwi K, Nyarko KM, Yarney J, Cox B. Cervical Cancer in the Greater Accra and Ashanti regions of Ghana. J Glob Oncol. 2017;3(6):782-90.

27. Yvonne Nartey PCH, Amo-Antwi K, Nyarko KM, Yarney J, Cox B. Factors contributing to the low survival among women with a diagnosis of invasive cervical Cancer in Ghana. Int J Gynecol Cancer. 2017;27:1926-34.

28. Musa J, Nankat J, Achenbach CJ, Shambe IH, Taiwo BO, Mandong B, Daru $\mathrm{PH}$, Murphy RL, Sagay AS. Cervical cancer survival in a resource-limited setting-north Central Nigeria. Infec Agents Cancer. 2016;11:15.

29. Odafe S, Torpey K, Khamofu H, Oladele E, Adedokun O, Chabikuli O, Mukaddas H, Usman Y, Aiyenigba B, Okoye M. Integrating cervical cancer screening with HIV care in a district hospital in Abuja, Nigeria. Niger Med J. 2013;54(3):176-84.

30. Chung MH, McKenzie KP, De Vuyst H, Richardson BA, Rana F, Pamnani R, Njoroge JW, Nyongesa-Malava E, Sakr SR, John-Stewart GC, et al. Comparing Papanicolau smear, visual inspection with acetic acid and human papillomavirus cervical cancer screening methods among HIVpositive women by immune status and antiretroviral therapy. AIDS. 2013;27(18):2909-19.

31. Engels EA, Biggar RJ, Hall HI, Cross H, Crutchfield A, Finch JL, Grigg R, Hylton T, Pawlish KS, McNeel TS, et al. Cancer risk in people infected with human immunodeficiency virus in the United States. Int J Cancer. 2008;123(1):187-94.

32. Joshi S, Muwonge R, Kulkarni V, Deodhar K, Mandolkar M, Lucas E, Sankaranarayanan R. Incidence of cervical intraepithelial neoplasia in women infected with human immunodeficiency virus (HIV) with no evidence of disease at baseline: results of a prospective cohort study with up to 6.4 years of follow-up from India. Int J Cancer. 2019;144(5):1082-91.

33. Landy R, Castanon A, Dudding N, Lim AW, Hollingworth A, Hamilton W, Sasieni PD. Cervical cytology and the diagnosis of cervical cancer in older women. J Med Screen. 2015;22(4):207-12.

34. Luhn P, Walker J, Schiffman M, Zuna RE, Dunn ST, Gold MA, Smith K, Mathews C, Allen RA, Zhang R, et al. The role of co-factors in the progression from human papillomavirus infection to cervical cancer. Gynecol Oncol. 2013;128(2):265-70.

35. International NPCNNal. Nigeria Demographic and Health Survey 2013. In: National Population Commission (NPC) [Nigeria] and ICF International. Abuja, Nigeria, and Rockville, Maryland, USA: NPC and ICF International; 2014.

36. Anorlu Rl. Cervical cancer: the sub-Saharan African perspective. Reprod Health Matters. 2008;16(32):41-9.

37. Yetimalar H, Kasap B, Cukurova K, Yildiz A, Keklik A, Soylu F. Cofactors in human papillomavirus infection and cervical carcinogenesis. Arch Gynecol Obstet. 2012;285(3):805-10.

38. Swaminathan R, Selvakumaran R, Vinodha J, Ferlay J, Sauvaget C, Esmy PO, Shanta V, Sankaranarayanan R. Education and cancer incidence in a rural population in South India. Cancer Epidemiol. 2009;33(2):89-93.

39. Smailyte G, Jasilionis D, Ambrozaitiene D, Stankuniene V. Educational inequalities in cancer incidence and mortality in Lithuania: a record linkage study. Cancer Epidemiol. 2012;36(5):e279-83.

40. Global Burden of Disease Cancer C, Fitzmaurice C, Allen C, Barber RM, Barregard L, Bhutta ZA, Brenner H, Dicker DJ, Chimed-Orchir O, Dandona R, et al. Global, Regional, and National Cancer Incidence, Mortality, Years of Life Lost, Years Lived With Disability, and Disability-Adjusted Life-years for 32 Cancer Groups, 1990 To 2015: a systematic analysis for the global burden of disease study. JAMA Oncol. 2017;3(4):524-48.

41. Ginsburg O, Bray F, Coleman MP, Vanderpuye V, Eniu A, Kotha SR, Sarker M, Huong TT, Allemani C, Dvaladze A, et al. The global burden of women's cancers: a grand challenge in global health. Lancet. 2017;389(10071):847-60.

42. Musa J, Achenbach CJ, O'Dwyer LC, Evans CT, McHugh M, Hou L, Simon MA, Murphy RL, Jordan N. Effect of cervical cancer education and provider recommendation for screening on screening rates: a systematic review and meta-analysis. PLoS One. 2017;12(9):e0183924.

\section{Publisher's Note}

Springer Nature remains neutral with regard to jurisdictional claims in published maps and institutional affiliations. 